\title{
DERECHOS EN PUGNA. DISPUTAS EN TORNO DE LA HERENCIA DE UNA SAYAÑA EN TERRITORIO AYMARA
}

\author{
RIGHTS IN CONFLICT. DISPUTES AROUND THE INHERITANCE \\ OF A SAYAÑA IN AYMARA TERRITORY
}

\author{
Alejandro Isla
}

\begin{abstract}
El artículo describe las prácticas de diferentes actores en un juicio centenario por una parcela en la Comunidad Originaria de Guaraya (hoy ayllu), lindera del pueblo de Tiwanaku, Bolivia. La parcela es una sayaña, un tipo de propiedad aymara que relaciona intereses colectivos (del ayllu) con intereses familiares, quienes la han ocupado, trabajado y realizado los rituales correspondientes durante generaciones. Así, el juicio revela lógicas individuales, de raigambre liberal, contrapuestas a colectivas, predominantes en la comunidad. Sin embargo, no abdican de disputar con sabiduría y astucia en espacios tribunalicios estatales, lejanos a su derecho consuetudinario. Los avatares del juicio litigioso - cuyas raíces se remontan a fines del siglo XIX-en tribunales nacionales de diferente competencia, ámbito geográfico, atravesando todas las instancias jurídicas, demuestran formas de microrresistencia, como de adaptación, en la construcción de autonomías comunitarias frente al Estado Nación, o en sus bordes. El texto se basa en la exégesis de un expediente judicial, orientada por el trabajo etnográfico del autor en Guaraya.
\end{abstract}

Palabras claves: antropología política, articulaciones Ayllu-Estado, microrresistencias, autonomía.

This article describes the practices of different actors in a 100-year long legal dispute for a plot in the Community of Guaraya (today ayllu), which adjoins the town of Tiwanaku in Bolivia. The plot is a sayaña, a type of Aymara property that relates (the ayllu's) collective interests with the interests offamilies, who have occupied and worked it, as well as performed the corresponding rituals in it for years. Thus, the trial reveals individual logics inspired by liberal traditions, which are in opposition to the collective ones prevalent in the community. These families, however, do not give up disputing with wisdom and ingenuity in State courts, which are alien to their customary law. The vicissitudes of the trial-whose roots go back to the late nineteenth century-in national courts of different jurisdictions, geographical scope, across all judicial instances, demonstrate forms of micro-resistance, as well as adaptation, in the construction of community autonomies before or in the margins of the Nation State. The text is based on the interpretation of a judicial file, guided by the author's ethnographic fieldwork in Guaraya.

Key words: Political anthropology, Ayllu-State interactions, micro-resistance, autonomy.

El litigio que presento describe la inexorable tendencia en la búsqueda de un camino autónomo de los ayllus aymara frente al Estado Nación boliviano, con obvias marchas y contramarchas a lo largo de su historia. Ese camino tuvo entre sus pilares fundamentales la lucha por recuperar y preservar sus antiguos territorios utilizando diferentes estrategias, según las oportunidades que aprovecharon de los resquicios de la dominación que por siglos sufrieron. En ese largo camino también debieron aprender -bajo coacción, negociación o su propio interés- códigos culturales diferentes a los propios, como el funcionamiento de instituciones ajenas.

Muchos de esos aspectos importados se han conformado con los propios en "sincretismos dinámicos”, más allá de la voluntad y consciencia de los actores involucrados en esos contactos fuertemente asimétricos respecto al poder alcanzado. Sincretismos que son resultado de la lucha de fuerzas hegemónicas. Fuerzas que no solo juegan en el campo moral y simbólico ${ }^{2}$, sino en las prácticas de producción y reproducción de la sociedad.

Así, mantuvieron instituciones o fragmentos de ellas (Albó 1972a, 1972b; Platt 1982), prácticas asociadas a las maneras de rotación del poder y tenencia de la tierra, como de la producción y distribución de los bienes producidos (Carter y Mamani 1982; Carter y Albó 1988; Isla 1992), que se mixturaron con formas coloniales de explotación y luego, con formas mercantiles y capitalistas en los altiplanos andinos a lo largo de los siglos XIX y XX. Todo ello, reforzado por una memoria

1 CONICET y FLACSO, Argentina. Caseros 453 - 3 piso B. CP 1152. Ciudad Autónoma de Buenos Aires, Argentina. lalewaira@gmail.com 
forjada en rituales y danzas, que actualiza el pasado, invocando el futuro (Abercrombie 1998; Saignes 1993a, 1993b).

Ello implicó profundas transformaciones en la cultura aymara, ya que en ese camino no tuvieron reparos en experimentar con el menú de alternativas que la modernidad propuso: lucharon por el reconocimiento sindical, por acceder a la educación formal, por su participación política local, parlamentaria y nacional (incluso mediante la construcción de partidos políticos de carácter étnico), probaron nuevas tecnologías agrarias, como la organización cooperativa ${ }^{3}$, llegando a la vicepresidencia de la República, como hoy a la presidencia.

También debieron aprender la lógica de las leyes que se les imponían, para conocer sus laberintos jurídicos. Ese ejercicio, que implicó resistencias y acomodaciones, no comenzó con la Revolución de 1952, sino que sus raíces se pierden en la historia colonial. En este artículo examino la complejidad de prácticas y estratagemas, de habilidades y perseverancias, por parte de comuneros aymara para litigar en un escenario jurídico que los situaba como subalternos. Un expediente del Consejo Nacional de Reforma Agraria ${ }^{4}$ expone una extendida querella por una parcela de tierra, descubriendo no solo un conflicto de poderes e intereses, sino lógicas normativas contrapuestas provenientes de tradiciones jurídicas distantes. También se pueden entrever pequeñas estafas y picardías de todos los involucrados. Prácticas que revelan clivajes étnicos y sociales, que Bolivia hereda de su pasado colonial.

La extendida disputa, que el caso ilumina, evidencia la tensión entre derechos consuetudinarios colectivos de posesión, sobre individuales. Impuestos éstos, bajo banderas del liberalismo en las tierras altas de Bolivia en los últimos casi cuarenta años del siglo XIX, perdurando su impulso en las primeras décadas del siglo XX. Los intentos espasmódicos que se sucedieron en ese período se encaminaron a dividir la tierra de comunidad, titulándola a nombre de propietarios, quienes de allí en más tributarían según el tamaño de sus parcelas. Agregando que la tierras que no estuvieran bajo título individual, eran confiscadas por el gobierno distribuyéndolas como latifundios entre allegados, de manera arbitraria y coactiva, con ingentes empleo de violencia. Hechos que fueron resistidos con desigual suerte usando un amplio menú de respuestas, como bloqueos de caminos, el control de la producción agropecuaria, las rebeliones agrarias que no escatimaron el uso de respuestas violentas, o empleando el pleito jurídico cuando las circunstancias políticas se volvían propicias, que discuto en este texto y que ha sido poco explorado en los estudios andinos.

El caso que trato echa luz sobre cuatro cuestiones, a saber:

(1) El parentesco aymara en relación a la tenencia y formas de propiedad de la tierra en una de las llamadas "comunidades libres". A su vez, revela conflictos inter e intrafamiliares por la propiedad.

(2) La participación de "vecinos del pueblo de españoles" de Tiwanaku, en estos litigios con el interés de adquirir una porción interesante de tierras cultivables con anterioridad a 1952/53.

(3) Los cambios de las leyes nacionales sobre propiedad y tenencia de la tierra (en la conformación del Estado-Nación boliviano) a lo largo del litigio, que pasan por varias transformaciones: de confección liberal en los procesos de Exvinculación a fines del siglo XIX (Demélas 2003) con intención de disolver derechos comunitarios en formas individuales, a un reconocimiento de incipientes formas colectivas; como fueron la cooperativa y el 'sindicato campesino'.

(4) Las transformaciones que introduce la Revolución de 1952 y su inmediata Reforma Agraria. Una de ellas, la participación creciente de la Comunidad como sujeto jurídico, en los escenarios tribunalicios nacionales. El expediente muestra una relación progresivamente más estrecha, de lo local con lo nacional. Pero ese proceso de acercamiento de la comunidad y el Estado, presenta una aparente paradoja: cuanto más crecen los vínculos entre ellos, más se afianza la autonomía y el poder del sujeto colectivo, frente al Estado-Nación.

El expediente del litigio, es el elemento central de las reflexiones que siguen. Sin embargo, para su análisis contextual e interpretación, retomaré resultados de la etnografía realizada años atrás. Es más, el expediente fue seleccionado en el Archivo General de la Reforma Agraria ya que refería directamente al cantón ${ }^{6}$ Tiwanaku donde comencé mis trabajos de campo en $1966^{7}$, y en particular a Guaraya, una comunidad que conocí con algún detalle (Figura 1). Aquellas etnografías y lecturas 


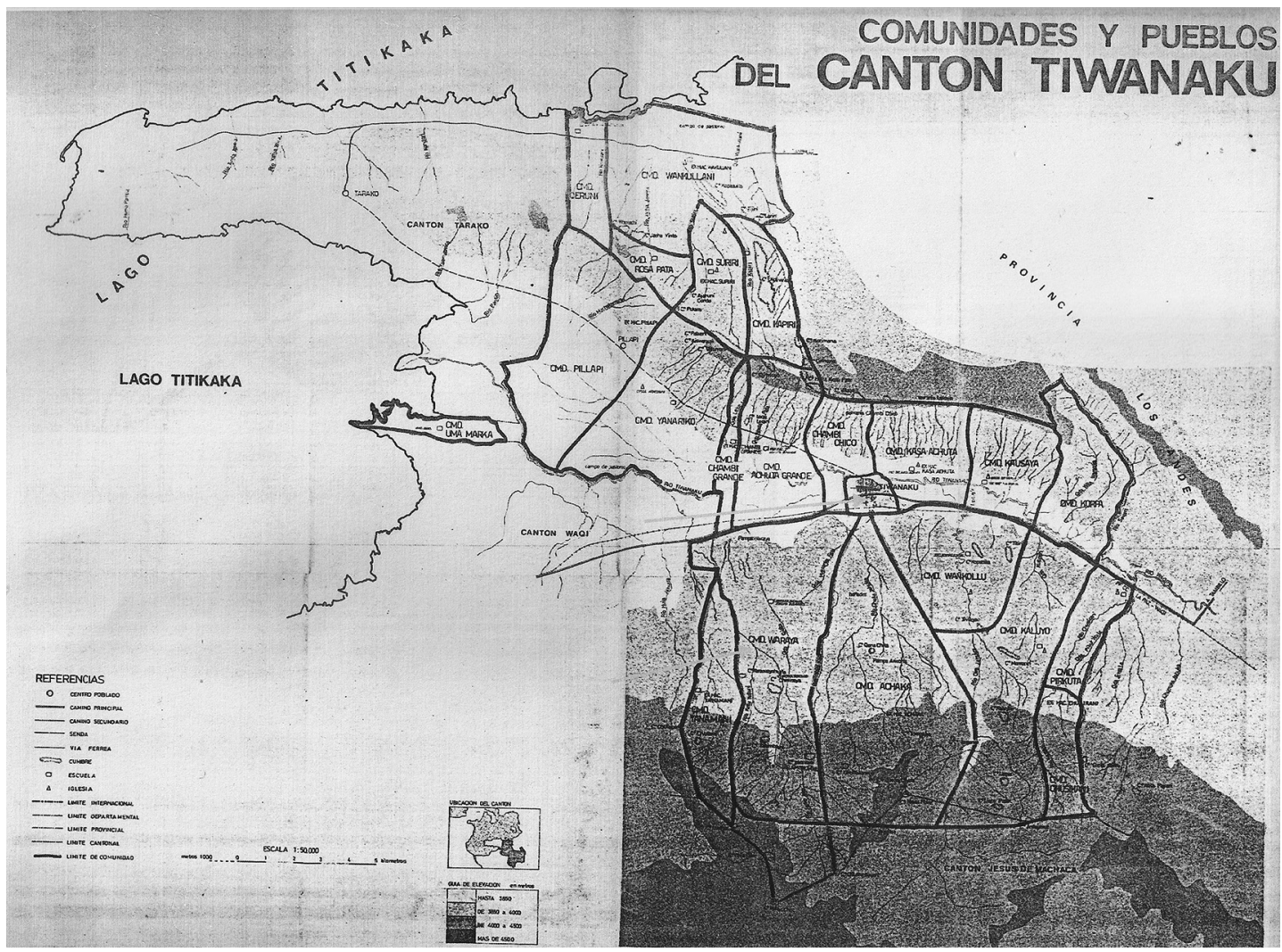

Figura 1. Mapa de cantón Tiwanaku y sus comunidades (confeccionado por el autor, 1985).

Map of the Tiwanaku canton and its communities (author's elaboration 1985).

complementarias, permiten las operaciones de análisis e interpretación del expediente de más de doscientas fojas, que obviamente resumo, intercalando comentarios y aclaraciones.

\section{El Escenario del Litigio}

El expediente se inicia en 1973 -con los ecos de la Revolución de 1952- pero los argumentos del juicio se remontan a fines del siglo XIX, con la presentación de escrituras, testimonios y la invocación de diferentes articulados. Sus actuaciones conclusivas (siempre apelables en litigios jurídicos ${ }^{8}$ ) se detienen a fines de los setenta del siglo XX a propósito de los cambios políticos que reconocían $\mathrm{y}$ anunciaban un poder autónomo creciente a comunidades, ayllus y/o sindicatos campesinos. En una trama básicamente de corte liberal, donde individuos invocan derechos, sobre propiedades, herencias, legitimidad de actos administrativos del pasado, y demás prerrogativas del derecho civil, se oculta en el fárrago de referencias a leyes, un actor colectivo que va inexorablemente desplegando su presencia, para confirmarse al final en el centro de la escena: la comunidad.

En el caso que trato, la parcela-de 28 hectáreas iniciales- que desata el conflicto es denominada sayaña en la mayor parte del escrito, lo que remite no solo a formas tradicionales de tenencia de la tierra en el mundo aymara, sino a un complejo campo de significaciones y creencias íntimas de cada familia, poseedora de sayaña. Allí reside un espíritu ancestral, que también respetan los demás miembros de la comunidad. Cada sayaña, no es simplemente una parcela, o un bien inmobiliario transable, cuya utilidad es la producción agropecuaria, por lo general irrigadas por canales construidos y mantenidos por los comuneros en jayma (trabajo obligatorio del conjunto). Cada sayaña es constitutiva de la familia, representante del linaje que allí reside. Conecta a los vivos que la ocupan y trabajan, con sus ancestros, con los espíritus de las montañas 
próximas, potentes y luminosas (las achachilas), pero también con el mundo oscuro de las cavernas.

A su vez, cada familia de sayaña tiene derechos en el territorio de la comunidad al usufructo de otras tierras esparcidas en su interior, estableciendo una red de solidaridades, conflictos e intereses que año a año se deben dirimir. Por ello, importantes miembros de la comunidad de sus diferentes zonas se avienen a participar como testigos en la justicia del Estado, dejando testimonio en el mamotreto. Unos comuneros apoyando a los descendientes del matrimonio Choque-Limachi y otros, otorgando cierta legitimidad a la compra de la sayaña por parte de Pascual Zuñagua.

La importancia del caso que ilumino se desarrolla en la Comunidad Libre de Guaraya, que no conoció el régimen de hacienda. Sin embargo, las Leyes de Exvinculación y las Revisitas que se sucedieron en la segunda mitad del siglo XIX y a principios del XX, tendieron $-\mathrm{y}$ en muchos casos lograron- a impulsar el régimen de hacienda en las tierras altas expulsando parte de sus habitantes, que debían reinstalarse en otros sitios; o bajo el régimen de la Hacienda quedaban como siervos o pongos; o intentando demarcar la tierra familiar de sayaña, para obtener tributos sobre las mismas (Rojas 1978). A principios del siglo XX la clase señorial advirtió que podían remplazar al indio en las yungas y tierras bajas del este, pero en las tierras altas de Puna era insustituible:

La exterminación simple de la raza indígena es el método heroico adoptado por los Estados Unidos (para solucionar el "problema indio")... Pero nosotros no podemos darnos ese lujo de los yanquis, porque el altiplano, expurgado de los indios, se convertiría en un yermo inhabitado e inhabitable (de Demetrio Canelas, Para los Terratenientes; en La Razón, La Paz, 21 de agosto de 1927; citado por Platt 1982:112)

De todas formas el predominio de la hacienda en las Tierras Altas, comenzó a extinguirse con la Revolución de 1952 que desató fuerzas más allá de sus ideales y objetivos de modernización. El caso que me ocupa, muestra cómo aymara rurales, además de responder con motines y rebeliones agrarias, bloqueos de caminos, disputaban palmo a palmo en el escenario judicial de Bolivia, distante del propio en el terreno conceptual, como moral.
No debería sorprender entonces, que en muchos lugares de las punas, próximas a la capital de Bolivia, hayan comenzado hace unos años a reinstalar el uso público -y legalizado por la nueva Constitución Nacional-del término ayllu para la forma comunidad; como marka para el pueblo centro de ayllus en su derredor. Sin embargo, en muchas de esas regiones el regreso a esos términos pre coloniales -mantenidos durante ese período y en gran parte de los tiempos de la república- no es contradictorio con el de Sindicato Campesino y Subcentral, para ayllu y marka respectivamente. Este cambio no fue solo una cuestión de maquillaje étnico, o de encerrarse en el pasado. Contrariamente al planteo de Crabtree y Chaplin (2012:48) que observan esas formas como opuestas, contradictorias y por tanto conflictivas entre sí, los aymara en su historia bajo dominación (que es la única que fehacientemente conocemos) han tenido la sabiduría de utilizar ideas, normas, productos, deidades, instituciones de sus dominadores, modelando en ellos estilos e improntas, mezclándolos con los propios. Antes que aquellos autores, Kusch (1973) desde Oruro, sostenía también de manera equívoca que elegían chayar a la Pacha para solicitar lluvias, antes que usar la bomba de agua para regar. La realidad es que imploraban a la Pacha por lo primero, pero también para que la bomba funcione. Muchos de los aspectos importados o impuestos, se han transformado con los propios en "sincretismos dinámicos", que se expresan en prácticas y formas organizativas, heterogéneamente mezcladas con las dominantes según las localidades. Como en el caso que trato aprendieron a moverse en espacios jurídicos conceptualmente distantes: entre sus normas consuetudinarias, procedimientos jurídicos coloniales, y los propios de la república.

\section{El Expediente Litigioso}

La causa se reactiva el 26 de octubre de 1973, a propósito de la presentación de un Testimonio (del 13 de febrero de 1959) efectuado por Pascual Zuñagua acompañado por una escritura de sesión de tierras. El Testimonio es encabezado por varios funcionarios:

que franquea el Secretario de Cámara del Consejo Nacional de Reforma Agraria, de varias piezas, dictadas dentro del proceso Agrario relativo al fundo denominado Caira Caraña $^{10}$, de cuyo tenor literal continua: 
Expediente 36.934. Fotocopia del título ejecutorial que corre a fojas diecisiete. Membrete de Hernán Siles Zuazo. Presidente Constitucional de la República ${ }^{11}$. Con Sello del Servicio Nacional de Reforma Agraria. Decreto Ley del 12 de Junio de 1954 (Serie U $74 \mathrm{~N}^{\circ} 160381-82^{12}$ ).

Iniciado en relación al fundo mencionado, el escrito pasa a su tratamiento con la denominación Pantipantini, distinta de la referida Caira Caraña. El objeto del Testimonio es la escritura de transferencia que otorga Zuñagua en favor de Luciano Toscanini. Esta escritura fue confeccionada por un "Notario Abogado", quien protocoliza en ese acto y da "Fé Pública", adjuntando a "...la minuta de compra venta de una sayaña rústica... un certificado de pago de impuestos fiscales ..." ( $\left.\mathrm{N}^{\mathrm{o}} 160390-83\right)$, trámite corriente para adquirir una propiedad inmobiliaria en la mayoría de los países de América, y uno de los resultados deseados por la Reforma Agraria: que las tierras subdivididas se inscriban en el Registro Nacional, a nombre de un propietario con fines impositivos. En la escritura de venta frente a notario, Zuñagua declara en su Clausula Tercera:

yo Pascual Zuñoaga, mayor de edad y hábil por derecho y excombatiente ${ }^{13}$, agricultor de la comunidad de Huaraya ${ }^{14}$, vecino del cantón Tiahuanacu, declaro que soy legítimo propietario y poseedor de la sayaña llamada Pantipantini de 28 has ... de la comunidad Huaraya ... provincia Ingavi del departamento de La Paz, el mismo que lo compré de su anterior propietario Carlos Choque y Manuela González de Choque, mediante escritura pública... ante el Notario Público... en fecha [16/ 11/ 1933] ${ }^{15}$ cuyo derecho de propiedad se haya inscrita en el Registro de Derechos Reales bajo la partida número... propiedad rústica que ha sido consolidada mediante título ejecutorial número... de [05/ 05/ 1960]... Al presente por así convenir a mis intereses, en forma libre y voluntaria sin que medie presión ni ningún vicio de consentimiento doy en venta real y enajenación perpetua los terrenos rústicos o sayaña Pantipantini antes referida con todas sus lihuas gallpas ${ }^{16}$, costumbres y servidumbres y usos ${ }^{17}$, sin limitación alguna, a favor del agricultor
Toscanini... la propiedad rústica que se transfiere no tiene gravamen hipotecario ni de ninguna clase, sin embargo el vendedor a salir a la evicción ${ }^{18}$ y saneamiento ${ }^{19}$ de Ley en caso necesario... ( $\left.\mathrm{N}^{\circ} 160390-83\right)$.

Entonces, Zuñagua había comprado la sayaña en 1933 y la inscribió recién en 1960 en el Registro de Bienes Reales. Está claro por esta clausula Tercera, que el comprador Toscanini se cubría de un posible uso del "instituto de evicción", derribando el acto de compra con el fin de "saneamiento" de los derechos sobre la sayaña. Y ese instituto fue solicitado por herederos legítimos de la misma en 1974. La Cuarta clausula de venta de1933, refiere:

mientras sea elevada la escritura pública, la presente minuta tendrá valor de documento privado y surtirá todos sus efectos jurídicos con simple reconocimiento de firmas y rubricas estampadas ( $\mathrm{N}^{\mathrm{o}}$ 160374-84). La Quinta refiere a la conformidad con la operación que da la esposa de Zuñoagua. La Sexta, estipula los límites de la 'propiedad rústica': ....colinda al Norte con el camino carretero La Paz-Desaguadero; al sud con la propiedad de Hugo Limachi... ( $\mathrm{N}^{\circ}$ 160374-84).

De todas formas, los Limachi -del linaje originario al que pertenecía la sayaña-demandantes en el expediente, aprovecharán la evicción del acto de venta que realizan sus herederos directos, refiriendo al Plano de la Sayaña Kayna Karaña, confeccionado en 1974, que abajo comento.

En la clausula Séptima del Testimonio, comienza la parte del comprador Toscanini, quien se declara

agricultor, ganadero, natural de Tiahuanacu... manifiesta su conformidad con todo lo expresado en el escrito. En el "pueblo de vecinos" 20 nombrado comparecen los actuarios a fin de rubricar sus firmas..." ( $\left.\mathrm{N}^{\circ} 160374-84\right)$. Al presente (08/ 11/ 1973) declaran vivir en Tiwanaku, apareciendo Zuñoagua como comerciante y Toscanini como agricultor, frente al Juez Parroquial de aquel pueblo. O sea, ambos no eran comuneros, vivían en el pueblo, aunque el primero laboraba la parcela en disputa ${ }^{21}$. 


\section{(a) Surgen los herederos}

Todo parecía convenientemente concluido según el Código Civil del Estado-Nación, cuando a continuación, en el expediente aparece otro Testimonio, que retrotrae las acciones a 1957. En el Expediente $\mathrm{N}^{\circ}$ 2324, adosado al documento y foliado, se consigna la presentación de Julia Pinedo viuda de Santos Choque, y sus hijos José y Manuel Choque, los legítimos herederos. Éstos demandan la

restitución de la sayaña de agregado 22 llamada 'PANTIPANTINI', ubicada dentro de la comunidad de Guaraya Grande... retenida por Suñagua desde el año 1935 de quien dice la compró a Petrona Limachi, abuela de los dos segundos demandantes y madre política de la primera mientras ellos se encontraban en la Compañía del Chaco... ( $\left.\mathrm{N}^{\circ} 160375-86\right)$.

Comunicado Zuñagua de la evicción, debe reiterar que compró la "propiedad rústica" a Carlos Choque y Manuela González de Choque en 1933, agregando que éstos la adquirieron de Luís Pando quien la obtuvo "en remate público" en $1926^{23}$. Requerida la opinión de los demandantes, manifiestan: “....asegurando ser falso las afirmaciones del demandado concluyendo en decir de que Suñagua usurpe la sayaña y se apropie violentamente, sin darle lugar a ellos que la posean...".

Frente a estas contradicciones flagrantes entre las partes involucradas, el Juez dispone distintos tipos de prueba. El primer tipo se denomina "Prueba Literal de los Actores". Demandantes y demandados deben aportar los escritos que corroboren sus dichos. La viuda de Choque y sus hijos presentan el título de Revisitas, cuya sola tenencia legitima su reclamo:

evidencia que en el cantón Tiahuanaco, provincia Pacajes (Ingavi) el señor Juez revisitador ${ }^{24} \ldots$ dividió en siete acciones las comunidades o ayllos de Huancollo, Achaca, Guaraya, Caboyo, Hinnamarca 25 y otros. Entre éstas Guaraya Grande y Guaraya Chico bajo las delimitaciones allí descritas... dispuso la adjudicación proindiviso ${ }^{26} \mathrm{de}$ dicha comunidad y consiguiente extensión del título correspondiente a sus poseedores, entre los que Apolinario Choque fue declarado propietario agregado y como consecuencia se le ministró posesión real, judicial y corporal, entre otros... (160376-87).

A continuación se inserta - como otra pruebauna declaratoria de herederos “.... a favor de Julia Pinedo esposa y supérstite de Santos Choque, y José y Manuel Choque hijos de éste..." mediante sentencia de 1956. Declaratoria que fue tramitada a los 33 años del fallecimiento de Santos Choque y a los 36 “... de la muerte del revisitario principal Apolinario Choque..." (160376-87). Sin embargo, esta sentencia fue declarada de 'ningún valor y nula', por los plazos dilatados -respecto a la fecha de los decesos- en los que fue presentada ${ }^{27}$. A fin de ilustrar el parentesco de los legítimos demandantes, y seguir las vicisitudes del litigio ver Figura 228 .

\section{(b) Solicitan pruebas testificales}

A renglón seguido de las pruebas 'Literales', continúan los testigos propuestos por los descendientes de Choque-Limachi:

declara el comunario Mariano Chambilla29, absolviendo los puntos del interrogatorio de fojas diecisiete dice: Ser cierto que el suegro de Julia Pinedo viuda de Choque, llamado Apolinar Choque, era propietario y matriculado de la sayaña 'Pantipantini' de agregado que se haya situada en la comunidad de Achajahuira ${ }^{30}$ de la jurisdicción de Tiahuanacu... ha quedado único heredero forzoso su hijo... esposo de Julia Pinedo, quedando su esposa sobreviviente... como heredera.

\section{Mariano agregará:}

que la sayaña no ha sido desajenada por los herederos, que en 1931 la viuda Petrona Limachi dio poder a Luís Pando para que defendiera su sayaña que se encontraba en litigio. Que éste despojó a la viuda de sus derechos y vendió la sayaña a Carlos Choque.

El segundo testigo Tomasa Bélice de Limachi, reitera lo dicho, agregando que Pando: 


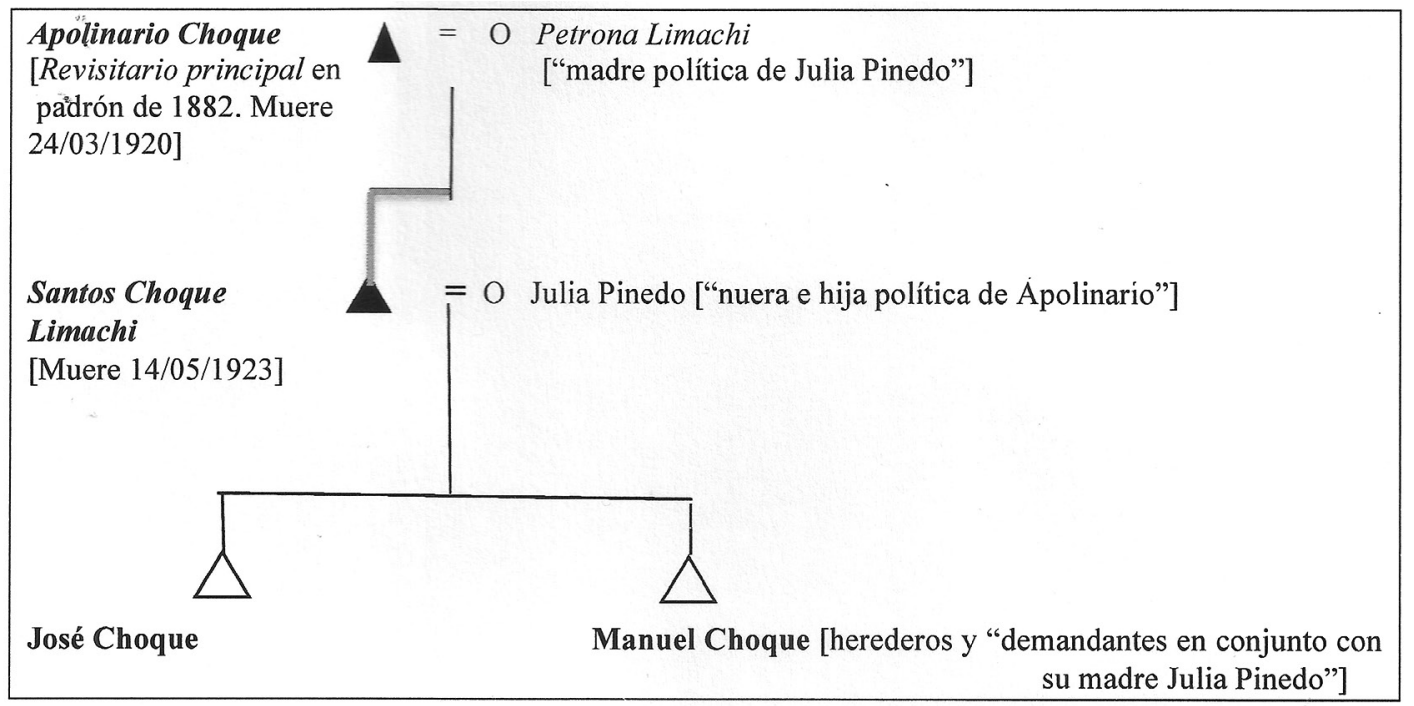

Figura 2. Diagrama de parentesco de los herederos legítimos. Kinship diagram of the legitimate heirs.

sin derecho alguno en la sayaña la vendió a Carlos Choque y este último por lo que no tenía papeles saneados y a toda súplica, Suñagua se hizo transferir la mencionada sayaña en calidad de venta... (160377-88).

O sea, se denuncia una doble estafa: un remate simulado y una venta fraguada. Dos testigos más por la demanda confirman estos testimonios.

A su turno Zuñagua presenta tres testigos -también de Guaraya- que por supuesto lo apoyan y contradicen los dichos anteriores. Sin embargo, el testimonio de Manuel Tonconi Chambillas destaca: declara "que Julia Pinedo viuda de Choque no ha estado en posesión ni sus hijos han trabajado labores agrícolas en la sayaña de agregado...". Luego, los herederos directos y demandantes de la evicción no laboraron la parcela, y ese hecho es conclusivo para normas de la cultura aymara, pesando en la resolución final.

Además, Zuñagua presenta la escritura pública de 1933 por la que Carlos Choque y esposa ${ }^{31}$-ante notario de la ciudad de La Paz-le venden la sayaña. En este punto es difícil seguir las maniobras que se tejen alrededor de la adquisición, remate o venta de Luís Pando. Quien asevera que consigue la parcela a partir de un "préstamo hipotecario", que le "sostiene" a la originaria Petrona Limachi, devolviendo el importe a un tercero con el dinero que "sacan" del remate ${ }^{32}$.
A partir de la Reforma Agraria y sus leyes posteriores (como la del 04/11/1955) la cuestión de permanecer en la tierra trabajándola fue decisivo para invocar su posesión. Pero además, la declaratoria de herederos no podía exceder los 30 años, y Pinedo y sus hijos, reconocidos herederos legítimos lo hicieron 33 años después de haber muerto Apolinario y su hijo Santos Choque ${ }^{33}$ : la declaratoria había prescrito, en relación a la 'restitución de la sayaña'.

\section{(c) De los fallos}

Invocando esas razones, el juez agrario de Guaqui, provincia de Ingavi, emite sentencia de primer grado a favor de Zuñagua en 1957. Fallo que se eleva al Tribunal Superior de La Paz, el cual analiza meticulosamente las controversias esgrimidas a lo largo de la historia, recorriendo el derecho que asiste a los actores involucrados.

En 1958 ese Tribunal considera que

los actores son descendientes directos del revisitario Apolinario Choque... declarado propietario de la sayaña Pantipantini el año 1882 en lo "proindiviso" 34 de la comunidad Guaraya Grande; empero de otro lado, también ha quedado demostrado que éstos no se hallan en posesión de la tierra resulta improcedente la restitución... que el demandado Pascual Zuñoaga deriva sus 
derechos propietarios desde la adquisición hecha por Luís Pando, en remate público el 08/05/1926... que aparte que el remate adolece de innumerables vicios que lo muestran como fraudulento ... en el caso de restitución muestra que intervino una de las partes copropietarias de la sayaña (la viuda Petrona Limachi)... y para tener validéz debía intervenir también Julia Pinedo y (sus dos hijos).

O sea, se niega la restitución de la parcela a los herederos pues no la trabajaban; pero al mismo tiempo se reconoce el derecho sucesorio legitimo que asiste a los mismos, y por tanto la “... afectabilidad de una cuota parte, al ser reconocidos como copropietarios de la sayaña ...", por lo que este tribunal superior, Sala Segunda del Consejo Nacional de Reforma Agraria “...confirma en parte la sentencia de primer grado... consolidando a favor del demandado (Zuñoaga) un (50\%) y el otro (50\%) se consolida a favor de los actores...", los herederos demandantes ( $\left.\mathrm{N}^{\circ} 202475-93\right)$. El conflicto parecía zanjado mediante un fallo que pretendía dejar conformes a todos los litigantes.

Sin embargo, al elevarse a su instancia máxima -al "Excelentísimo Señor Presidente Constitucional de la República, a través del Ministerio de Asuntos Campesinos", La Paz 1959-el curso del litigio cambia radicalmente de rumbo al reconocerse un tercer 'actor' que había permanecido activo pero en las sombras de los tribunales, como del expediente: la comunidad, o el ayllu como se califica en la actualidad.

El fallo de Siles reconocía “...que la sayaña de autos, si bien fue de comunidad, se transfirió en remate público en 1926, estando actualmente trabajada por el demandado (Zuñoaga)...", si bien el veredicto anterior asumió en partes iguales los derechos de los herederos como el del comprador, “...los comuneros de Guaraya reclaman esa injustificada concesión del $50 \%$... ya que quedarían despojados de sus liguas, que por costumbres establecidas, poseen en lo proindiviso y en forma rotativa todos los comunarios, para cultivos de cierto tiempo..." [mi énfasis]. O sea, aparece explícitamente la comunidad de Guaraya como colectivo, y que justamente por sus fueros reclama legitimidad en su demanda. Entonces el fallo de 1976-firmado por el presidente de Bolivia-resuelve que el $50 \%$ de la sayaña que había sido asignado a los herederos se consolida a favor de la comunidad, correspondiéndole a aquellos una indemnización legal, pues además de no trabajar esa tierra, vivían y trabajaban en La Paz.

Por el lado de Zuñagua, la cuestión se complica aún más al recuperar -en la resolución de 1976- la comprobación realizada en 1959 sobre el registro de compra, el cual antes de ser protocolizado confirmaba que “....sufrió adulteración por medio de raspaduras en la columna que se consigna el nombre ${ }^{35}$ así como también en la que corresponde a la superficie... que el $50 \%$ asignado a la familia Choque se 'consolida'... con sus liguas a favor de la comunidad Guaraya..." (94-95). La falsificación es denunciada para el "enjuiciamiento criminal" por la apoderada de la viuda Rita Limachi, encabezando la nómina de familiares ${ }^{36}$ (Figura 3) ${ }^{37}$.

\section{Conclusiones}

El litigio por la tierra de sayaña que se desarrolla a lo largo de tres generaciones desde 1882 hasta 1976, revela cómo los aymara rurales intentaban, y a veces alcanzaban resultados positivos, en un mundo extraño a sus lógicas jurídicas consuetudinarias. El derecho de sustento liberal enarboló como estandarte la "propiedad privada e individual" de la tierra, atacando las lógicas colectivas andinas en aras de introducir y consolidar "la civilización" desde mediados del siglo XIX, mediante las Leyes de Exvinculación. Frente a ello los aymara apelaron a las más diversas estrategias para conservar territorio y autonomía. Con la Reforma Agraria de 1953 se introdujeron 'formas colectivas' de formato Occidental, como el sindicato campesino y la cooperativa, para reemplazar haciendas, pero también a ayllus que habían subsistido. Sin embargo, en gran parte de las tierras altas, dispositivos o aspectos de aquellos formatos modernizantes, fueron ensamblados y sincretizados en antiguas relaciones sociales comuneras de producción y distribución manteniendo prácticas asamblearias y rotación de cargos.

Una cuestión a destacar es que el conflicto muestra varios intereses en pugna. Zuñagua debe probar la legitimidad de la compra de las 28 ha en 1923, como de su venta a Toscanini en 1959. Los Choque herederos, quieren recuperarla para revenderla ellos, ya que nunca las trabajaron y vivían en La Paz. Toscanini vecino de Tiwanaku -que conocía parte de los entuertos de aquellas operaciones- intenta quedarse con la totalidad de derechos que guarda una sayaña en la cultura 


\section{PLANO DE LA SAYAÑA \\ KAYÑA RARAÑA \\ COMUNIDAD: GUARAYA}

Cant. TIAHUANACOPROV. INGAVI

\section{Depto. LA PAZ}

ESCALA $7: 5.000$

Sup. 21,3000 Has.

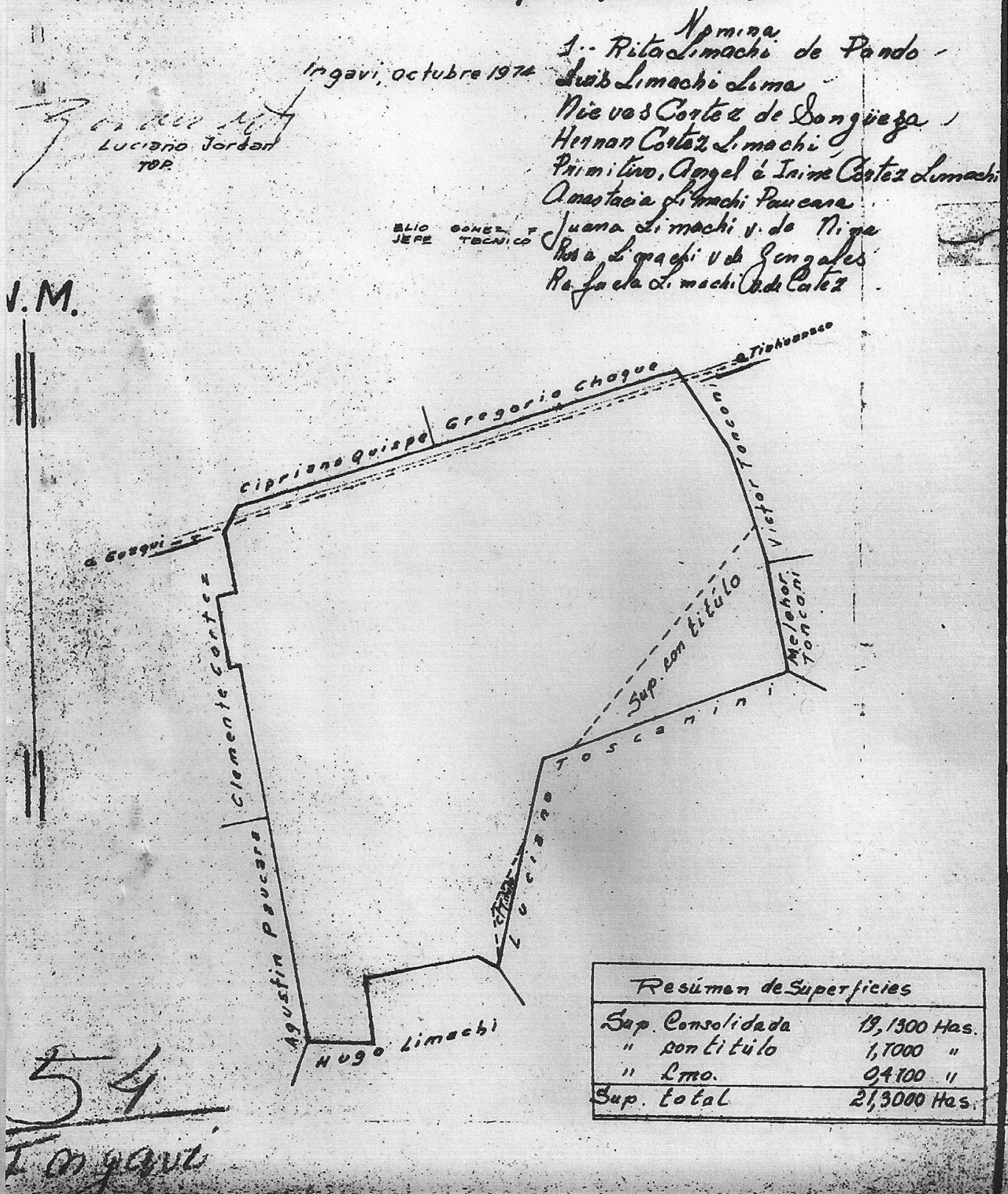

Figura 3. Plano de la sayaña en litigio [Sito en expediente].

Plan of the sayaña in dispute [In legal file]. 
aymara. Por fin la comunidad, representada por los Limachi, parientes directos por línea materna de Petrona, "originaria" de Guaraya, retoma el control del territorio. Pero también Petrona, no recurre a su nuera y nietos -quienes poseían el título de la sayaña de agregado, y por ello doblemente legítimos herederos- para la venta simulada mediante remate público. Queda la sospecha si Petrona es estafada por Pando, o en realidad fue su cómplice en la maniobra. Por ello, la cuestión étnica -'aborígenes' aymara vs. 'vecinos' de Tiwanaku- no alcanza para entender la disputa.

La vía de la disputa jurídica, necesitó conjugar astucia, tenacidad, paciencia y empeño en un paulatino conocimiento de leyes, decretos, y fundamentalmente las lógicas y procedimientos del Estado republicano. Esas cualidades no solo se demuestran con el resultado final, sino con la presencia del "ayllu" a lo largo del expediente, por medio del reconocimiento explícito de un tipo particular de propiedad aymara como la sayaña, que liga un linaje a un territorio, como a un conjunto de valores constitutivos de las relaciones sociales en esa cultura.

Para ello los aymara bolivianos, se movieron en lo que denominé una aparente paradoja: para conservar o aumentar su poder subalterno, no debían aislarse -como persiguió el Estado colonial y luego el republicano, a lo largo de siglos- sino intentar conocer la lógica de leyes que los subordinaba, comenzando con el aprendizaje del castellano, los mecanismos que ordenaban los intercambios comerciales y más tarde, el reclamo de la educación formal, sin abandonar gran parte de su cultura y territorios.

Agradecimientos: En memoria de don Mariano Chambilla. A los comuneros de Guaraya. Agradezco especialmente las críticas, comentarios y sugerencias que realizaron al texto los evaluadores propuestos por Chungara.

\section{Referencias Citadas}

Abercrombie, T. 1998. Pathways of Memory and power. Ethnography and History among and Andean People. The University of Wisconsin Press, Wisconsin.

Albó, X. 1972a. Esposos, Suegros y Padrinos entre los Aymara. CIPCA, La Paz.

Albó, X. 1972b. Dinámica de la estructura intercomunitaria de Jesús de Machaca. América Indígena 32(3):773-816.

Ayllu Guaraya Originario 2013. Estatuto Comunal. En www. ftierra.org (20/04/2017) Fundación Tierra, Bolivia.

Carter, W. y X. Albó 1988. La comunidad Aymara: un mini-estado en conflicto. En Raíces de AMÉRICA. El Mundo AYMARA, editado por X. Albó, pp. 451-493. Alianza América/ UNESCO, Madrid.

Carter, W. y M. Mamani 1982. Irpa Chico. Individuo y Comunidad en la Cultura Aymara. Editorial Juventud, Bolivia.

Choque, R. y X. Albo 2003. Cinco Siglos de Historia. Jesús de Machaqa, la Marka Rebelde. CIPCA, La Paz.

Comaroff, J. y J.L. Comaroff 1991. OfRevelation and Revolution. Christianity, Colonialism, and Consciousness in South Africa. The University of Chicago Press, Chicago.

Crabtree, J. y A. Chaplin 2012. Bolivia: Processes of Change. Zed Books, London.

Creham, K. 2002. Gramsci, Culture and Anthropology. Pluto Press, London.

Demélas, M.D. 2003. Attaques et résistances. Les communautés indiennes en Bolivie au XIX ${ }^{\mathrm{e}}$ Siècle. En Les Propiétés Collectives face aux Attaques Libérales (1750-1914). Europe Occidentale et Amérique Latine, editado por M.D. Demélas y N. Vivier, pp. 303-323. Presses Universitaires de Rennes, Rennes.
De Munter, K. y T. Salman 2009. Extending political participation and citizenship: Pluricultural civil practices in contemporary Bolivia. Journal of Latin American and Caribbean Anthropology $14: 432-456$

Gisbert, T. 1992. Los curacas del Collao y la conformación de la cultura mestiza andina. Senri Ethnological Studies 33:52-102.

Gramsci, A.1971. Letteratura e vita Nazionale. Quaderni del Carcere. Editori Riuniti, Roma.

Henáiz, I. y D. Pacheco 2000. La Ley INRA en el Espejo de la Historia. Dos Siglos de Reformas Agrarias en Bolivia. Fundación Tierra, La Paz.

INRA (Instituto Nacional de Reforma Agraria) 2008. Breve Historia del Reparto de Tierras en Bolivia. De la Titulación Colonial a la Reconducción Comunitaria de la Reforma Agraria: Certezas y Proyecciones. La Paz.

Isla, A. 1984. Sistemi per l'adattamento socioculturale nelle terre alte a Sud del Lago Titicaca. Studi per l'Ecologia del Quaternario 6:103-124.

Isla, A. 1992. Dos regiones, un origen. Entre el "Silencio y la Furia”. En Sociedad y Articulación en las Tierras Altas Jujeñas. Crisis Terminal de un Modelo de Desarrollo, compilado por A. Isla, pp. 167-217. ECIRA- ASAL, Buenos Aires.

Kusch, R. 1973 El Pensamiento Indígena y Popular en América. Editora Ica. Buenos Aires.

Platt, T. 1982. Estado Boliviano y Ayllu Andino. Instituto de Estudios Peruanos, Lima.

Rivera Cusicanqui, S., E. Schwartzberg Arteaga, P. Mamani Ramírez, H. Pruden, M. Geidel, H. Castaño Silva, T. Abercrombie, B. Arzáns de Orsua y Vela, Á. Pinaya Pérez, J.J. Vaca Carraffa, 
L. Alemán Vargas, P. Quisbert, G. Behoteguy Chávez, V. Auza Aramayo y G. Fernández Juárez 2010. Principio Potosí Reverso. Museo Nacional de Arte Reina Sofía, Madrid.

Rojas, A. 1978. La tierra y el trabajo en la articulación de la economía campesina con la hacienda. Avances 2:51-70.

Saignes, T. 1993a. Estar en otra cabeza. Tomar en los Andes. En Borrachera y Memoria. La Experiencia de lo Sagrado en los Andes, editado por T. Saignes, pp. 11-23. Hisbol/IFEA, La Paz.
Saignes, T. 1993b. Borracheras Andinas. ¿Porqué los indios ebrios hablan en español? En Borrachera y Memoria. La Experiencia de lo Sagrado en los Andes, editado por T. Saignes, pp. 43-73. Hisbol / IFEA, La Paz.

Spedding, A. 2015. Catre de Fierro. Editorial Plural, La Paz.

Zavaleta Mercado, R. 1986. Lo Nacional-Popular en Bolivia. Siglo XXI, México.

\section{Notas}

1 Concepto inspirado en Gramsci (1971:268).

2 Comaroff y Comaroff (1991:29) en sus estudios postcoloniales, equiparan "ideología" con "hegemonía" en Gramsci, interpretando aquella con lo dicho y explícito, y la hegemonía con lo inconsciente e inscrito, en lo que llaman la "cadena de concientización". Dentro de la antropología, pero desde otra postura teórica, Crehan (2002:174) critica su posición desde una lectura de los Cuadernos de la Prisión más atenta, insistiendo que la hegemonía para Gramsci, “... always involves 'practical activity', and the social relations that produce inequality, as well as the idea by which that inequality is justified, explained, normalized....". Eludiendo el debate sobre el concepto de "hegemonía", rescato el énfasis de Crehan sobre las 'actividades prácticas', pero también el papel de lo simbólico en la conformación del "sentido común".

3 Dándoles impronta propia, a las que perduran en un ayllu.

4 Sito en la Secretaría de Cámara (Sala "A") en La Paz, engordado con diferentes instrumentos probatorios.

5 Así denominadas porque no sufrieron el régimen de la Hacienda antes de la Reforma Agraria de 1953. En el excantón Tiwanaku existieron dos: Chambi y Guaraya.

6 Actualmente municipio.

7 Continué en 1968. Luego volví al campo desde fines de 1984 hasta principios de 1986.

8 Los fallos resolutivos en conflictos de tierras son provisorios cuando existen cambios radicales en los marcos jurídicos, como en el acceso a derechos de poblaciones que permanecieron por siglos en las sombras del Estado-Nación, sin reconocimiento de ciudadanía. Para un análisis profundo de la conformación boliviana, Zabaleta Mercado (1986). Para una reconsideración del concepto de ciudadanía en la cultura aymara contemporánea, De Munter y Salman (2009).

9 Sobre el tema existe para las culturas de Andes Centrales excelentes trabajos -realizados desde diferentes ópticas disciplinarias y estilos de representación-que refieren a una diversidad de manifestaciones: pintura, escultura, grabados, bailes, ceremonias, el lenguaje, costumbres cotidianas, etc. Entre los últimos trabajos recomendaría el espléndido montaje de video, poesía, foto, danza, antropología e historia que realiza Rivera Cusicanqui et al. (2010). También, la notable novela de Spedding (2015) que hecha luz sobre la mezclas del español con el aymara en el lenguaje cotidiano paceño; cuestión que se transpone al mundo de creencias y valores y que atraviesa la historia boliviana y a las clases sociales que la componen. Y por supuesto la erudición y el ojo de Gisbert (1992), con sus revelaciones sobre la Escuela Cuzqueña y la formación de la cultura mestiza.

10 Lo que va entre comillas es copia fiel del expediente.

11 En su primer mandato presidencial de 1956 a 1960.

12 El expediente presenta un doble foliado, uno impreso Serie U $74 \mathrm{~N}^{\circ} 160381$ y otro manual: 82 en este caso; en adelante cito: $\mathrm{N}^{\mathrm{o}} 160381-82$.

13 Combatiente en la Guerra entre Bolivia y Paraguay [193235], quienes tuvieron mención honorífica.

14 Guaraya, como hoy escriben sus comuneros.

15 En el documento, las fechas en letras.

16 'liwa qalpa', nombre aymara de pequeñas parcelas esparcidas en todo el territorio de la comunidad, utilizadas para la agricultura como complemento de la sayaña, y que elabora un complejo entretejido entre los intereses de las familias (Carter y Mamani 1982; Isla 1984, 1992).

17 Una fórmula proveniente del derecho colonial. Refiere a las 'servidumbres de paso' (caminos o senderos que atraviesan la parcela), y al 'uso y costumbre', sobre por ejemplo los canales de riego y los turnos de acceso al agua.

18 Pérdida de un derecho por sentencia firme, en virtud de derecho anterior ajeno. La 'evicción' proviene del derecho romano, y nutrió el derecho español. Continua vigente en Bolivia con la republica. El agregado precautorio indicado, responsabiliza al vendedor, augurando el futuro desenlace.

19 El riesgo de una sentencia judicial o administrativa, derivada de una acción reivindicatoria anterior al título de adquisición del vendedor, en base a derechos alegados por terceros; como sucedió.

20 Los moradores de Tiwanaku lo definían en 1966 como 'pueblo de 'vecinos' o 'de españoles', agregando despreciativamente 'no de indios que viven en los campos'. Pero también vivían aymaras, algunos en matrimonios mixtos.

21 El escrito termina certificando el pago de transferencia, timbres, actas a la "Administración Distrital (colecturía) del pueblo de Laja"; en camino a Tiwanaku a $35 \mathrm{~km}$ de La Paz. Por fin, se consigna que la escritura queda rubricada en La Paz por un "Notario de Fe Pública" el 22 de enero de 1974 y se "inscribe" 15 de abril de 1974 en esa capital por el Juez Registrador de Derechos Reales.

22 El agregado, Apolinario Choque, no era originario de Guaraya, aunque aymara de otra comunidad del cantón Tiwanaku. Adquiere la sayaña en la revisita de 1882, casándose con Petrona Limachi, originaria de Guaraya. 
23 Un "remate público" resulta sospechoso para la época en una comunidad aymara.

24 No se trataba de una usurpación descarada y violenta como en la época de Melgarejo de1864 a 1871 (Demélas 2003). Las revisitas consistían “....en una usurpación protegida por leyes, decretos, resoluciones que se fueron dictando durante quince años. Se originaron extensos latifundios, en los que el indio fue reducido a servidumbre (pongo), aunque no perdió la esperanza de reivindicar lo que considera hasta el presente como herencia de sus antepasados" (INRA 2008:14).

25 Las comunidades de Caboyo e Hinnamarca no existen al día de hoy. Las tres primeras han perdurado. Wancollo y Achaka estuvieron bajo el régimen de hacienda hasta 1953. Guaraya, siempre "comunidad libre", para 1966 conservaba el apelativo de Guaraya Grande, veinte años después, sus comuneros y autoridades revirtieron la divisoria creada en 1882 por el Juez revisitador Acosta: Chica y Grande se reunificaron como Guaraya.

26 Una persona ostenta el derecho de propiedad sobre un bien solo parcialmente: lo comparte con otras personas. Es el caso de los ayllus o comunidades aymaras.

27 En la época, declaratorias de herederos, testimonios, etc. eran desconocidos para los aymara rurales. La litigiosidad se ensanchó y complejizó post Reforma Agraria donde el Código Civil boliviano sufrió varias enmiendas. Pero más allá que los aymara, quichuas y otros originarios eran discriminados y excluidos de la ciudadanía como parte de las poblaciones subalternas, se les hacía imposible acceder con solvencia a la letra de la ley, sospechada de imparcialidad. La propia idea y plazos de prescripción de una causa les eran ajenos.

28 En negro, los fallecidos al realizarse la escritura de venta a Zuñoaga en1933.

29 Hace medio siglo Don Mariano y su familia, me hospedaron. Combatió en la guerra del Chaco, cayendo prisionero de los paraguayos; de quienes conservaba buenos recuerdos. Fue comando del MNR en la Revolución del 52.

30 Don Mariano sostiene que Apolinario Choque “...era propietario y matriculado de la sayaña Pantipantini de agregado situada en la comunidad de Achajahuira de la jurisdicción de Tiahuanaco..."; no he encontrado rastros de esa comunidad en registros referentes a Tiwanaku. Quizá fuera convertida en hacienda a fines del XIX, como lo fue el territorio de la actual Comunidad Centro Wakullani en la 'zona norte' del ex cantón Tiwanaku. Su territorio es surcado por el río Jacha Jahuira, [río grande, o potente], cuya grafía se asemeja a como los escribientes llevaron a papel los dichos de don Mariano. Wakullani fue una -en en la costa del Titicaca- de las numerosas propiedades de don Gabriel Fernández Guarachi -importante cacique de Pakajes- que al morir en 1681 abre una sucesión conflictiva entre sus dos hijas legítimas (Choque y Albó 2003:89-96).

31 Quienes la adquirieron de manera fraudulenta de Pando.

32 Petrona había obtenido un crédito anticrético de 600 bolivianos otorgado por Rita Belis. Petrona no logra devolver el préstamo en término, solicitando ayuda de Pando. Éste parodia un remate, adjudicándose la sayaña. Luego simula una venta, paga aquella deuda, y probablemente se queda con algún dinero restante. Se menciona en el expediente una causa penal contra Pando.

33 En los folios se cita la fecha de muerte de suegro y marido, no la de su suegra Petrona Limachi -"originaria” de Guarayaque muere bastante más tarde. Se estaba considerando aún los derechos sucesorios por línea paterna, para determinar la prescripción.

34 O sea, el Tribunal superior reconoce que por ser sayana es parte de la comunidad.

35 De la sayaña: borran el nombre original Kayña Karaña, y ponen Pantipantini. Ésta solo ocupaba una parte de aquella. Además, denuncian a Zuñoaga y Toscanini de haber tergiversado el título ejecutorial entre Zuñoaga y la "Comunidad de Huaraya". Por estas reiteradas y graves alteraciones, producidas en diferentes períodos, se demanda que "...se constituya en el terreno una comisión precedida por el Juez Agrario e integrada por el topógrafo correspondiente a objeto que proceda en derecho a la verificación de los confines de la propiedad de los mandantes..." (Figura 3).

36 Transcribo la "Nómina" que figura en el plano de la Figura 3. Rita Limachi de Pando; Luís Limachi Lima; Nieves Cortez de Sangüeza; Hernan Cortez Limachi; Primitivo, Angel é Inine Cortez Limachi; Anastacia Limachi Paucara; Juana Limachi viuda de Nina; Rosa Limachi viuda de González; Rafaela Limachi de Cortez. En itálica, Rosa Limachi quien figura en el Estatuto del Ayllu Guarayas originario del 2013.

37 Cuando parecía el litigio definitivamente cerrado, Toscanini objeta mediante una -"excepción perentoria de cosa juzgada"- parte del levantamiento topográfico sosteniendo “...que los demandantes se han propasado en su sayaña denominada Pantipantini...". La fracción reclamada por éste queda consignada en el vértice derecho inferior del plano (Figura 3). Los resquemores subsisten hasta el presente, a pesar que los derechos comunales se han consolidado. 